ISSN: $1130-3743$

\title{
PANTALLAS Y EDUCACIÓN: ADOLESCENTES Y VIDEOJUEGOS EN EL PAÍS VASCO
}

\author{
Screens and education: teenagers and videogames \\ in the Basque Country
}

\author{
Écrans et éducation: jeux vidéo et adolescents \\ au Pays Basque
}

José Ignacio Imaz BengoetXea

Universidad del País Vasco. Facultad de Filosofía y Ciencias de la Educación.

Departamento de Teoría e Historia de la Educación. Avenida Tolosa, 70.

20018 San Sebastián, Guipúzcoa. Correo-e: j.imaz@ehu.es

Fecha de recepción: enero de 2011

Fecha de aceptación definitiva: abril de 2011

Biblid [(1130-3743) 23, 1-2011, 181-200]

\section{RESUMEN}

El artículo recoge los principales resultados de una investigación empírica dedicada al análisis de la relación "videojuegos-educación" entre adolescentes vascos. La investigación ha tenido dos objetivos: realizar una primera aproximación cuantitativa al uso de juegos digitales entre los adolescentes vascos y diseñar y probar talleres para analizar la posible utilización educativa de los juegos electrónicos. Los datos de la encuesta indican que la mayoría de ellos se autodefine como usuario de juegos digitales, y que no parece haber diferencias significativas entre jugadores y no jugadores en cuanto a hábitos de ocio y uso de Tic. En la segunda parte de la investigación, se han realizado cuatro talleres o experimentos. Las sesiones han mostrado que los juegos electrónicos pueden ser interesantes materiales de apoyo en la escuela.

Palabras clave: tecnología educativa, influencia de la tecnología, educación secundaria. 


\section{SUMMARY}

This article brings together the main results of an empirical study dedicated to analysing the relations between videogames and education in the Basque Country. The research had two major aims. First, it set out to produce an initial quantitative estimate of the use of digital games among Basque adolescents. The second goal was to design workshops to test and analyse the possible educational uses of videogames. The data show that the majority define themselves as videogames users and that there does not appear to be any significant difference between players and non-players. In the second part of the study, we tested four workshops or experiments. The sessions have shown that the electronic games can be interesting support material at the schools, and that the motivation of the students is very high in this kind of proposals.

Key words: educational technology, influence of technology, secondary education.

\section{SOMMAIRE}

Cet article recueille les principaux résultats d'une recherche empirique sur l'analyse du rapport "videojeux-education" parmi les adolescents basques. La recherche a les suivants objectifs: 1 , Mise en ouvre d'une première approche quantitative sur l'utilisation des jeux digitaux parmi les adolescentes basques; 2, dessiner et tester des ateliers pour analyser la possible utilisation éducative des jeux électroniques. Les donnés de l'enquête nous montrent que la plupart des adolescents se reconnaît comme usagers des jeux digitaux et qu'il ne semble pas avoir des différences significatives entre joueurs et non-joueurs. Dans la deuxième partie de la recherche, on a mis en ouvre quatre ateliers ou expérimentes. Les sessions ont montré que les jeux électroniques peuvent être des intéressants matériaux d'appui à l'école et que la motivation des élèves est très haute face á ce tipe de propositions.

Mots clés: technologie éducative, influence de la technologie, éducation secondaire.

\section{INTRODUCCIÓN}

La cultura digital y las pantallas son elementos centrales de las sociedades postindustriales y postmodernas de la información. Dentro de esta cultura, los videojuegos (o "juegos electrónicos o digitales") han adquirido en los últimos años una gran relevancia social, cultural y económica. Se trata de la industria del ocio que más dinero recauda, superando al cine y a la música. En 2008, el valor de las ventas de la industria del videojuego en Europa (juegos y consolas) fue de 15.000 millones de euros, un 15\% más que el año anterior. España aparece en el cuarto puesto del ranking europeo, por detrás de Reino Unido, Alemania y Francia, con 1.454 millones de euros gastados en el 2008, lo que supone un 57\% del consumo audiovisual (Adese, 2010). 
El 25\% de la población de la Unión Europea y el 22,5\% de la población española (10,4 millones de personas) se declara "jugador habitual de videojuegos", cifra que asciende al 60\% cuando nos referimos a los adolescentes entre 11 y 18 años (Adese, 2009).

Sin desdeñar los aspectos polémicos del fenómeno que también hay que analizar y explicar ${ }^{1}$, el presente artículo presenta las conclusiones de una investigación que ha tenido otros objetivos: en primer lugar, realizar una aproximación cuantitativa para analizar el uso de los juegos electrónicos entre los adolescentes vascos; en segundo lugar, diseñar y probar una propuesta para trabajar con los videojuegos desde un punto de vista educativo ${ }^{2}$.

\section{MARCO TEÓRICO: REPASO DE LOS ESTUDiOS MÁS IMPORTANTES SOBRE JUEGOS DiGITALES Y EDUCACIÓN}

Son numerosas las investigaciones que en los últimos años han tratado de demostrar las potencialidades educativas de los videojuegos. Para James Paul Gee, por ejemplo, los buenos videojuegos se basan en interesantes principios educativos: aprendizaje activo y mayor posibilidad de interacción con el medio que en cualquier otro formato (literatura, cine, televisión...); posibilidad de probar diferentes roles, de

1. Dos de los problemas con los que se asocia habitualmente a los videojuegos son por ejemplo la violencia y el sexismo. Entre los autores y las instituciones que creen que hay que tomarse muy en serio el problema de la violencia en los videojuegos podríamos destacar a AMNisTía InTERnACiONAL (2004), Anderson, Gentile y Buchley (2007), Byron (2008), Etxeberría (2006 y 2008), Grossman y Degaetano (1999), Levis (1997), Mandres (2008). Otros autores, sin embargo, creen que se está creando una excesiva alarma social: De Miguel (2006), Estallo (1995), Freedman (2002), Jones (2002), Kutner y Olson (2008). Para analizar la relación entre los videojuegos y el sexismo, ver: Provenzo (1991), CASSEL y JENKINS (1998), Díez GutiérRez (2004). En relación con estos aspectos polémicos, nos parecen interesantes las respuestas que plantea David Willianson Shaffer (Universidad de Wisconsin-Madison, EE. uu.) en su página web a algunas de las preguntas más frecuentes: "Sí, existen muchos videojuegos que pueden ser perjudiciales para los niños, así como existen muchos programas de televisión y muchos libros que pueden ser perjudiciales para los niños. Tendríamos que saber diferenciar las cosas, y tendríamos que tener claro que muchos juegos digitales no son apropiados para menores [...]. Por lo tanto, los educadores deberían tener información y educación sobre este tema, para ayudar a los niños a elegir los juegos apropiados [...]. Y sí, la gente puede caer en la adicción con cualquier cosa que le guste. Los menores deberían mantener un equilibrio entre los diferentes tipos de actividades que realizan: leer, ejercicio y actividad física, manualidades... Pero eso no supone que se les tengan que prohibir los juegos electrónicos" (www.epistemicgames.org, descargado el 17 del 10 de 2009).

2. La investigación ha sido financiada por la Universidad del País Vasco (Código OTRI NUPV 06/17) y la Sociedad de Estudios Vascos (Ayudas a la Investigación, Convocatoria 2007), y se ha terminado de escribir en el Centre for Cultural Policy Research (СCPR) de la Universidad de Glasgow, gracias a una estancia de investigación en calidad de Visiting Fellow en el marco de un acuerdo de colaboración entre el CCPR y el Departamento de Educación del Gobierno Vasco. Se ha coordinado el estudio (con la ayuda de Félix Etxeberría) y han participado como colaboradores Garbiñe Bereziartua, Daniel Artamendi, Aitor Urigüen y Mikel Miralles. 
conocer diferentes contextos, de simular diferentes situaciones (sin el peligro y los costes económicos que supondría probar en la realidad algunas de esas situaciones, como por ejemplo pilotar aviones); variedad y riqueza de formatos: imagen, sonido, textos; feed-back constante; fuerte identificación con los personajes y las historias; utilización de la imaginación, la fantasía, el juego... (Gee, 2004, 2007).

En opinión de Henry Jenkins, no podemos pensar una buena educación para el siglo xxi sin tener en cuenta los medios digitales. La nueva cultura y sociedad de la información exige nuevas competencias, y son necesarias políticas educativas para esta alfabetización digital: en primer lugar, porque sin políticas educativas públicas, las desigualdades socioeconómicas pueden suponer una desigualdad de oportunidades en el acceso a estas nuevas experiencias, capacidades y conocimientos ("el problema de la igualdad de oportunidades"). En segundo lugar, porque sin intervención pedagógica podrían crearse problemas para entender cómo condicionan e influyen los nuevos medios nuestra visión del mundo ("el problema de la trasparencia"). En tercer lugar, porque como todas las generaciones, también las nuevas generaciones que vivirán en la sociedad digital deben ser educadas con criterios morales y éticos ("el reto ético"). Por lo tanto, la educación es necesaria también en la nueva cultura: para ofrecer igualdad de oportunidades a todas las personas, para ayudar a entender los medios digitales y para fijar las bases éticas (Jenkins, 2006).

Para Javier Echeverría, las familias y los sistemas educativos deberían tomarse muy en serio el fenómeno de los videojuegos, porque se trata de los juegos del "tercer entorno" (el mundo digital), y los juegos son elementos muy importantes en cualquier cultura. Así como se aprende a vivir en el primer (la naturaleza) y en el segundo entorno (la ciudad), viviendo y jugando en esos mundos, los videojuegos son los juegos del tercer entorno, los juegos que nos enseñan a vivir en el espacio electrónico. Los militares ${ }^{3}$ y los empresarios ${ }^{4}$ ya han entendido las posibilidades de los videojuegos, y ahora es necesario por lo menos un mínimo de participación del sector público en la gestión y el control de este sector, porque de lo contrario estaríamos dejando la cibersociedad y la cibercultura en manos del mercado:

Desde el punto de vista pedagógico, aprender jugando es uno de los lemas clásicos de la pedagogía. Hoy en día hay que ampliar dicho lema a los videojuegos, introduciéndolos en las escuelas y centros de formación. Los procesos de e-learning no sólo han de centrarse en las aulas electrónicas, también han de tener los correspondientes e-patios, es decir, lugares donde los niños y niñas dispongan de un amplio elenco de videojuegos, previamente seleccionados por los educadores y centros escolares [...] Promover una cultura de la innovación en los ámbitos educativos

3. Ver el juego de propaganda del Ejército de Estados Unidos en americasarmy.com.

4. Página web de la patronal española del sector de los videojuegos: adese.es (Asociación de Distribuidores y Editores del Software de Entretenimiento). 
requiere fomentar el uso de algunos videojuegos, seleccionándolos en función de la edad, las capacidades y los valores (Echeverría, 2008, 12).

Son varias las organizaciones que están utilizando videojuegos con fines educativos. La onu ha lanzado por ejemplo Food-force y Against the odds con el objetivo de concienciar a las nuevas generaciones sobre problemas como "el hambre en el mundo" y "la situación de los refugiados". UnICEF, por su parte, ha presentado los juegos Stop disasters (para prevenir desastres naturales), Ungefanyaje (¿Tú que harias?, en suahili, para informar sobre el SIDA) y Planeta Ozonalia (para promover el consumo responsable).

Cada vez podemos encontrar más "videojuegos serios", "videojuegos políticosociales" o "videojuegos con conciencia", e incluso se ha celebrado el "Primer congreso de Videojuegos para el Cambio" (Nueva York, junio del 2006). Allí se presentaron juegos como Peacemaker (basado en el conflicto Palestina-Israel), pero existen muchos más: A force more poweful (activismo a favor de la democracia, la paz y los derechos humanos), Organizing Game (activismo político), Darfur is dying (crisis humanitaria de Darfur), Pax Warrior (genocidio de Ruanda), Palestina y Latinoamérica ${ }^{5}$ (trabajo didáctico sobre conflictos mundiales), I can end deportation (crítica de la política de inmigración de Estados Unidos), Refugee (concienciación sobre la situación de los refugiados), El mundo de Dina (derechos de la infancia), Antiwargame (crítica de la guerra)... La empresa vasca Ikasplay dedicada a los "videojuegos serios" y a los "simuladores didácticos" ha recibido en febrero del 2011 el Premio a la Joven Empresa Emprendedora de España (iasplay. com). Interesantes colectivos independientes están utilizando también videojuegos: Bordergames en Madrid, Ravalgames en Barcelona, Molleindustria (Italia-Estados Unidos), News Gaming...

Es interesante también el trabajo que está realizando en el ámbito de los «juegos y mundos virtuales serios" el Serious Games Institute (Universidad de Coventry, Reino Unido). El instituto analiza cómo pueden utilizarse los juegos digitales y los mundos virtuales con objetivos "serios" (negocios, turismo, educación...), y organiza diferentes congresos y conferencias sobre este tema: Serious Virtual Worlds Conferences, International Conferences in Games and Virtual Worlds for Serious Aplications $^{6}$ (seriousgamesinstitute.co.uk). Entre sus proyectos podemos encontrar, por ejemplo, el denominado EduTeams, que se probó durante el año 2005 en varios colegios de la ciudad escocesa de Dundee con muy buenos resultados. El objetivo del proyecto era trabajar con videojuegos habilidades como el «trabajo en

5. Los juegos Palestina y Latinoamérica han sido diseñados por la compañía danesa Serious Games (seriousgames.dk, globalconflicts.eu). El director de la compañía, Simon Egenfeldt-Nielsen, también ha publicado dos libros sobre la materia (EgENFELDT-NielSEN, 2007 y EgENFELDT-NieLSEN, SMITH y Pajares Tosca, 2008).

6. Entre los congresos dedicados al estudio de la relación entre juegos digitales y educación habría que destacar también las European Conferences on Games Based Learning (ECGBL). 
equipo" y la "comunicación y el liderazgo" con alumnos de entre 10 y 14 años. Los juegos para el proyecto fueron diseñados expresamente por la interesante empresa Team Play Learning Dynamics (tpld.net), especializada en diseñar juegos digitales para uso educativo. Para quienes quieran profundizar en este campo, es interesante también el estudio publicado en el 2008 por la actual directora del instituto, Sara de Freitas: Serious Virtual Worlds: a scoping study. En el mismo, De Freitas explica cómo podrían los mundos virtuales utilizarse con objetivos serios, a través de varios estudios de caso. Por ejemplo, la plataforma Active Worlds nos ofrece la posibilidad de crear nuestro propio mundo virtual, o de comprar uno ya diseñado. Dentro de este proyecto general, el espacio Active Worlds Educational Universe ha sido especialmente diseñado para el ámbito educativo, y ya está siendo utilizado por diversas universidades. De Freitas explica en su informe cómo se ha utilizado la plataforma en la Educación Primaria de Singapur, para enseñar ciencias a alumnos de 11 años con malos resultados escolares. En ocho sesiones de dos horas cada una, los alumnos construyeron virtualmente el sistema solar, con una mejora evidente en los resultados, tanto en cuanto al aprendizaje de contenidos como en cuanto a motivación y actitud. Otro estudio de caso lo constituye el uso de la plataforma Forterra's OLIVE (Online Interactive Virtual Environment) por parte de la Facultad de Medicina de la Universidad de Stanford (California, Estados Unidos): utilizando la plataforma se ha diseñado virtualmente la Entrada de Emergencias de la Facultad y se han preparado diferentes casos para que los alumnos aprendan a realizar correctos diagnósticos. En la famosa red social virtual Second Life podemos encontrar el espacio SciLands, el espacio para la ciencia y la tecnología en el que participan 23 organizaciones dedicadas al estudio de este campo (De Freitas, 2008).

El grupo que lidera David Willianson Shaffer en la Universidad de WisconsinMadison (eE. uu.) trabaja en una línea de investigación parecida (Shaffer, 2008). Para Shaffer, si queremos utilizar los juegos digitales en la educación, lo primero que deberíamos saber es que no nos sirve cualquier juego. Los juegos que interesan a este grupo de investigación son los que imitan "los trabajos de los adultos", los "roles profesionales", juegos que ellos han definido como epistemic games. Estos "juegos de conocimiento" son juegos que enseñan al jugador la forma de pensar necesaria para vivir en el mundo digital. Juegos que promueven capacidades como la creatividad y la innovación, muy importantes en la sociedad en la que nos ha tocado vivir. Y para ello han diseñado expresamente algunos juegos (epistemicgames.org): en el videojuego Escher's World, el jugador se convierte en un artista gráfico que debe preparar una exposición utilizando la geometría y el diseño gráfico; en el videojuego Pandora Project, el jugador participa en algunos de los debates médicos más importantes del momento, para lo cual deberá aprender biología, genética, ética, etc.; en el juego journalism.net nos convertimos en periodistas, y en Science.net en periodistas científicos que tras entrevistar a expertos y recopilar información deben preparar reportajes (los trabajos que se preparan para estos dos últimos juegos se cuelgan luego en la Red). 
Autores como Marc Prensky, sin embargo, sí creen en la utilidad educativa de cualquier videojuego, incluso en la de los juegos comerciales más polémicos. Para Prensky, juegos como, por ejemplo, los de la saga Grand Theft Auto (en los que el jugador debe robar coches, repartir palizas, etc.) sirven para plantear interesantes debates: ¿Qué son y para qué sirven las normas y las leyes? ¿Quién debería ocuparse de su cumplimiento? ¿Cómo? ¿Debería el gobierno controlar este tipo de videojuegos? ¿Debemos ser críticos con este tipo de juegos? ¿Por qué? (Prensky 2001, 2002, 2006) ${ }^{7}$.

En España, el grupo pionero y seguramente más importante en la utilización de videojuegos comerciales con fines educativos ha sido F9 (Gros, 1998, 2004, 2008), pero son interesantes también el trabajo del "Grupo Imágenes, Palabras e Ideas" (GIPI) de la Universidad de Alcalá de Henares (Lacasa et al., 2007a, 2007b, 2009) y el proyecto SPIDER (Smarter People Through Interactive Digital Entertainment Resources) dirigido desde la Universitat Oberta de Catalunya por Daniel Aranda y Jordi Sánchez-Navarro ${ }^{8}$. En cuanto a números monográficos de revistas científicas, podríamos destacar dos de Comunicación y Pedagogía ("Los videojuegos en la escuela" del año 2000 y "Utilización didáctica de los videojuegos" del 2006) y otro de Teoría de la Educación: Educación y Cultura en la Sociedad de la Información ("Videojuegos: una herramienta en el proceso educativo del Homo Digitalis" del 2008).

Kurt Squire, en su tesis doctoral presentada en la Universidad de Indiana (EE. Uu.) analizó cómo podía utilizarse el videojuego Civilization III para aprender historia. Podemos consultar las conclusiones de esta investigación en la página web gameslearningsociety.org (página del grupo de investigación que dirige actualmente K. Squire: Games, Learning and Society en la Universidad de WisconsinMadison, EE. UU.): tutoriales para aprender a jugar, guion para trabajar diferentes periodos históricos basándonos en los capítulos del juego, actividades complementarias (preguntas y referencias a fuentes de información), etc.

Es también muy interesante el proyecto Brain Meld (Bridging the gap between gaming and education), liderado por Ted Henning desde la National University, School of Media and Communication (California, Estados Unidos). Nos gustaría destacar especialmente las teaching guides o fichas didácticas, porque en nuestra opinión pueden ser muy útiles para aquellos que quieran trabajar los videojuegos desde una perspectiva crítica y reflexiva. Organizadas por edades (se proponen diferentes juegos para diferentes edades), las fichas recogen información muy concreta sobre lo que se puede hacer desde un punto de vista educativo con los

7. Página web de M. Prensky en la que podemos encontrar "fichas didácticas" para trabajar educativamente los videojuegos y consejos para profesores y padres: gamesparentsteachers.com.

8. Página web de estos tres grupos: xtec.es/ abernat (F9), http://www2.uah.es/gipi (GIPI) y http://spider-uoc.blogspot.com (SPIDER). 
videojuegos: qué objetivos del currículum oficial se trabajarían con cada juego, esquema concreto de cada sesión, cómo se debe jugar... (brainmeld.org)?

\section{OBjeTIVOS Y PlANTEAMIENTO METODOLÓGICO DE LA INVESTIGACIÓN}

El objetivo de nuestra investigación ha sido analizar la relación entre juegos digitales y educación. Este objetivo general se ha concretado en dos objetivos específicos:

- Objetivo "sociológico": plantear una primera aproximación cuantitativa al fenómeno para medir el uso de juegos electrónicos entre los adolescentes vascos.

- Objetivo "pedagógico": analizar cómo se pueden trabajar y aprovechar estos nuevos juegos desde un punto de vista educativo.

A la hora de realizar el diseño metodológico de la investigación, el primer objetivo exigía claramente la utilización de la encuesta para la recogida de datos, aunque, teniendo en cuenta los recursos disponibles, nos hemos tenido que limitar a realizar una primera aproximación que no es estadísticamente representativa.

433 adolescentes de entre 11 y 18 años contestaron nuestra encuesta en junio del 2008 en cinco colegios de la Comunidad Autónoma Vasca. Las preguntas principales que estructuraron el cuestionario fueron: ¿Cuánto se utilizan los videojuegos? ¿Cuál es la frecuencia de juego y durante cuánto tiempo se juega? ¿Cuáles son los juegos favoritos? ¿Qué relación existe entre los juegos digitales, las actividades de ocio y el uso de TIC?

En la muestra se han tenido en cuenta la variable género, el tipo de escuela (públicas y privadas-concertadas) y la presencia en las tres provincias de la Comunidad Autónoma Vasca (Tabla 1). Siendo conscientes del carácter exploratorio de este estudio y de las limitaciones de la muestra en cuanto a tamaño y representatividad

9. Otras propuestas de fichas didácticas para trabajar con videojuegos: AmNISTía INTERNACIONAL (2004): Videojuegos: ¿qué valores transmiten? (i-a.es) y la ficha que acompaña al juego Contra viento $y$ marea (contravientoymarea.org). Entre las experiencias prácticas que ya funcionan en este campo nos gustaría destacar el proyecto del Departamento de Educación del Gobierno de Escocia, Bringing the Computer Games into the Classroom (ltscotland.org.uk/gamesbasedlearning). Uno de los responsables de este proyecto, Derek Robertson, tiene un blog interesante sobre el uso de videojuegos en la escuela: hotmilkydrink.typepard.com. Otros blogs de profesores que están trabajando con videojuegos en sus clases: timrylands.com (Tim Rylands, Escocia), McDivitt.wordpress.com (Dake McDivitt, EE. Uu.), mackenty.org (Bill Mackenty, EE. uu.). También desde Escocia, desde la Universidad Heryot Watt de Edimburgo, se lidera el proyecto europeo Ecircus, con el objetivo de analizar la posible utilización de juegos de rol virtuales para la educación social y emocional (e-circus.org). En el Reino Unido también han empezado a utilizar videojuegos los alumnos con necesidades especiales (Ellis, Heppell, Kirriemuir, Krotoski y McFarlane, 2006; Sandford y Williamson, 2005). Es también muy interesante la investigación Games in Schools de la European Schoolnet (hhtp://games.eun.org). En el marco de este proyecto se ha publicado Videojuegos en el aula. Manual para docentes (2009). 
de la misma, las conclusiones del presente trabajo, a pesar de que no son estadísticamente generalizables, sí presentan evidentes tendencias; que indican, a su vez, líneas futuras de investigación con tamaños muestrales que permitan disminuir los errores inferenciales, a la vez que posibiliten la globalización de los resultados.

TABla 1. Caracterí́sticas De la MUESTRA ${ }^{10}$

\begin{tabular}{|c|c|c|c|}
\hline & Alumnos de escuelas públicas & Alumnos de escuelas privadas & Total \\
\hline Chicos & 133 & 76 & 209 \\
\hline Chicas & 135 & 89 & 224 \\
\hline Total & 268 & 165 & 433 \\
\hline
\end{tabular}

Para el segundo objetivo, hemos diseñado y probado talleres para trabajar los juegos electrónicos en las escuelas. La recogida de datos se ha realizado utilizando las técnicas de la observación participante y el cuestionario semiestructurado. Se eligieron un colegio público y otro privado-concertado. En las sesiones han participado 96 adolescentes ( 50 chicas y 46 chicos). Las cuatro sesiones se llevaron a cabo en otoño del 2008, las dos primeras en la escuela Urola de Azpeitia y las otras dos en la escuela Laskorain de Tolosa, ambas en la provincia de Gipuzkoa.

\section{LA PARTE CUANTITATIVA-SOCIOLÓGICA DE LA INVESTIGACIÓN: LA ENCUESTA}

La mayoría de los adolescentes que han contestado la encuesta, casi un 80\% $(79,1)$, utiliza los videojuegos, dato que coincide con el del resto de estudios realizados en países de la OCDE, situándose por debajo de la media de Estados Unidos, donde el 95\% de los adolescentes entre 12 y 17 se declaran jugadores (Kane, Middaugh y Evans, 2008), y por encima de la media española, que no llega al 70\% (Adese, 2009; Rodríguez, 2002; Camas y Almazán, 2006; Ferrer y Ruiz, 2005). Si nos fijamos en los chicos, la media sube al 97\%, mientras que "sólo" el 62\% de las chicas ha contestado que utiliza los videojuegos, lo que viene a confirmar también las diferencias de género que se observan en otras investigaciones (diferencias que se están reduciendo en los últimos años). En cuanto a la edad, como se puede apreciar en el Gráfico 1, son los más jóvenes los que más juegan (el 100\% de los que tienen 11 años), y el uso desciende a medida que se hacen mayores.

10. Al no tratarse de una muestra aleatoria, no se presentan los índices de representatividad. Los cinco colegios que participaron en la encuesta fueron: Laskorain (Tolosa, Gipuzkoa), KarmeloEtxegarai (Azpeitia, Gipuzkoa), Gurutzeta (Baracaldo, Bizkaia), Ballonti (Portugalete, Bizkaia) y Miguel de Cervantes (Vitoria, Álava). Tenemos que agradecer la ayuda prestada por el profesor Juan Etxeberría (Universidad del País Vasco, Facultad de Filosofía y Ciencias de la Educación, Departamento de Métodos de Investigación y Diagnóstico en Educación) a la hora de diseñar la encuesta y analizar los datos. 


\section{GRÁFICO 1}

USO DE VIDEOJUEGOS ENTRE LOS ADOLESCENTES ENCUESTADOS (2008)

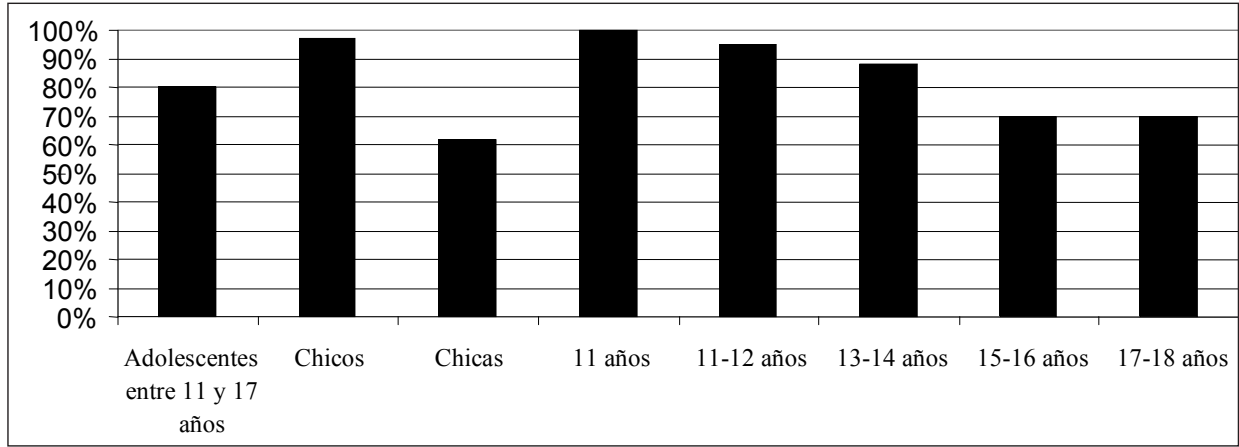

En cuanto a la frecuencia de juego (Tabla 2), las respuestas se distribuyen de forma regular entre los que juegan una vez al mes, los que juegan una vez a la semana, los que juegan dos o tres veces por semana y los que juegan todos los días. Pero como en la mayoría de las preguntas, existen diferencias significativas entre chicos y chicas, porque los chicos juegan más. Y también la edad es una variable discriminadora, siendo los más jóvenes los que más juegan (aunque hay más “jugadores diarios» entre los de 18 años que entre los de 11).

TABLA 2

Frecuencia de uso de los VIDEOJUEgos (PORCENTAJE SOBRE LOS QUE HAN CONTESTADO LA ENCUESTA)

\begin{tabular}{|c|c|c|c|c|c|}
\cline { 2 - 6 } \multicolumn{1}{c|}{} & Una vez al mes & Una vez a la semana & $2-3$ veces/semana & Todos los días & \\
\hline Chicas & 47,7 & 25,8 & 16,1 & 10,3 & 100 \\
\hline Chicos & 11,1 & 22,8 & 37,9 & 28,2 & 100 \\
\hline 11 años & 17,1 & 19,5 & 48,8 & 14,6 & 100 \\
\hline 18 años & 40,5 & 4,8 & 26,2 & 28,6 & 100 \\
\hline Total & 27 & 24 & 28,7 & 20,4 & 100 \\
\hline
\end{tabular}

A la pregunta de cuánto juegan cada vez (Tabla 3), la mayoría contesta 0-2 horas, dato que también coincide con el resto de investigaciones (Adese, 2009). Los días festivos, un grupo numeroso juega entre dos y cuatro horas, y un grupo pequeño pero significativo, más de cuatro horas (el 90\% de este grupo son chicos). 
TABLA 3

¿CUÁNTO SE JUEGA?

(\% SOBRE LOS QUE HAN CONTESTADO LA ENCUESTA)

\begin{tabular}{|l|c|c|c|c|}
\cline { 2 - 5 } \multicolumn{1}{c|}{} & $0-2$ horas diarias & $2-4$ horas al día & 4 horas diarias & \\
\hline Días escolares & 96,3 & 3,4 & 0,3 & 100 \\
\hline Días festivos & 61,5 & 28,8 & 9,8 & 100 \\
\hline
\end{tabular}

En la pregunta "¿cuál es tu videojuego favorito?", el juego más citado fue Pro Evolution Soccer, uno de los simuladores de fútbol más conocidos que encajaría en la tipología de "deportes". En segundo lugar aparece Grand Theft Auto (GTA), que podríamos definir sobre todo como juego de "acción-aventura", pero que toca también otros géneros (simulación, lucha, estrategia, carreras...). En este caso es también destacable el hecho de que el segundo videojuego favorito de los adolescentes de entre 11 y 17 años sea un juego recomendado para mayores de 18 años. La lista continúa con los siguientes nombres: Mario Bros (plataforma-laberinto), Sims (simulación), FIFA (fútbol), NBA (baloncesto), Need for Speed (carreras), Sing Star (simulación-música), Gran Turismo (carreras), F1 (carreras), Pressing Catch, Tekken, Counter Strike, Medal of Honor, wwe, Call of Duty, Dragon Ball, Good of War (lucha-guerra-tiro), Eye Toy Play, Wii Sports (deportes)... En esta pregunta habría que subrayar la amplia dispersión de respuestas, porque los encuestados han citado más de 100 videojuegos diferentes. No obstante, cabe destacar que son los mismos juegos que se citan en el resto de países de Europa, en Estados Unidos, en Japón... (con unas poquísimas excepciones para referencias a algunos juegos de la Televisión Vasca o de la Asociación de Escuelas Vascas). Es decir, los videojuegos parecen erigirse en un claro ejemplo que viene a confirmar la teoría del peligro de homogenización cultural en el nuevo mundo globalizado. En el País Vasco se juegan los mismos juegos que en el resto de países industriales o postindustriales.

En la última parte de la encuesta analizamos las posibles diferencias entre jugadores y no jugadores en cuestiones como "hábitos de ocio" y "uso de TiC».

TABLA 4.1

¿QuÉ HaCES EN TU TIEMPO LIBRE? (\% SOBRE LOS QUE HAN CONTESTADO LA ENCUESTA)

\begin{tabular}{|l|c|c|c|c|c|c|}
\hline & \multicolumn{3}{|c|}{ DEPORTE } & \multicolumn{3}{c|}{ ESTAR CON LOS AMIGOS } \\
\hline & $\begin{array}{c}\text { No jugadores } \\
\text { (NO-J) }\end{array}$ & $\begin{array}{c}\text { Jugadores } \\
(\mathrm{J})\end{array}$ & $\begin{array}{c}\text { Los que juegan } \\
\text { mucho (JM) }\end{array}$ & No-J & $\mathrm{J}$ & $\mathrm{JM}$ \\
\hline Nunca & 10,1 & 4,1 & 2,9 & & 0,6 & \\
\hline Pocas veces & 21,5 & 15,1 & 5,7 & & 3 & \\
\hline A veces & 34,2 & 29,3 & 34,3 & 16,5 & 22,8 & 31,4 \\
\hline Muchas veces & 34,2 & 51,5 & 57,1 & 83,5 & 73,7 & 68,6 \\
\hline & 100 & 100 & 100 & 100 & 100 & 100 \\
\hline
\end{tabular}


TABLA 4.2

¿QUÉ HACES EN TU TIEMPO LIBRE? (\% SOBRE LOS QUE HAN CONTESTADO LA ENCUESTA)

\begin{tabular}{|l|c|c|c|c|c|c|}
\hline & \multicolumn{3}{|c|}{ LEER } & \multicolumn{3}{c|}{ ESTAR DELANTE DEL ORDENADOR } \\
\hline & No-J & $\mathrm{J}$ & $\mathrm{JM}$ & No-J & $\mathrm{J}$ & $\mathrm{JM}$ \\
\hline Nunca & 15,9 & 22,6 & 25,7 & 1,2 & 6 & 2,9 \\
\hline Pocas veces & 41,5 & 42,3 & 42,9 & 12,3 & 22,9 & 5,7 \\
\hline A veces & 35,4 & 28 & 22,9 & 42 & 35,8 & 40 \\
\hline Muchas veces & 7,3 & 7,1 & 8,6 & 44,4 & 35,2 & 51,4 \\
\hline & 100 & 100 & 100 & 100 & $100 \%$ & 100 \\
\hline
\end{tabular}

En lo que respecta al ocio (Tablas 4.1 y 4.2), los jugadores dicen hacer más deporte y utilizar menos el ordenador que los no jugadores (datos que no concuerdan con el estereotipo con el que normalmente se define a los videojugadores), pero, por otra parte, suelen pasar menos tiempo con los amigos y leen menos (datos que sí confirmarían el estereotipo, aunque las diferencias no son demasiado grandes).

En cuanto al análisis del uso de las nuevas tecnologías (Tablas 5.1 y 5.2), nuestra encuesta tampoco confirma la imagen estereotipada del jugador de juegos digitales que se ha construido en las últimas décadas: el videojugador no es un "friki" enganchado a las nuevas tecnologías, no al menos más enganchado que cualquier otro adolescente de su misma edad (con algunas pocas excepciones: por ejemplo, los que juegan mucho utilizan Internet más que los demás).

TABla 5.1. ¿CuÁNTO utilizas las SigUiENTES teCnOlogías? (\% SOBRE LOS QUE HAN CONTESTADO LA ENCUESTA)

\begin{tabular}{|l|c|c|c|c|c|c|}
\hline & \multicolumn{4}{|c|}{} & \multicolumn{2}{c|}{$\begin{array}{c}\text { MONTAR VIDEOS Y COLGARLOS } \\
\text { EN SITIOS COMO Youtube }\end{array}$} \\
\hline & $\begin{array}{c}\text { No jugadores } \\
\text { (No-J) }\end{array}$ & Jugadores (J) & $\begin{array}{c}\text { Los que juegan } \\
\text { mucho (JM) }\end{array}$ & No-J & $\mathrm{J}$ & $\mathrm{JM}$ \\
\hline Nunca & & 3,8 & & 33,3 & 38,5 & 25,7 \\
\hline Pocas veces & 4,8 & 10,6 & 5,7 & 21 & 19,7 & 25,7 \\
\hline A veces & 27,4 & 22 & 11,4 & 32,1 & 21,8 & 25,7 \\
\hline Muchas veces & 67,9 & 63,6 & 82,4 & 13,6 & 20 & 22,9 \\
\hline & 100 & 100 & 100 & 100 & 100 & 100 \\
\hline
\end{tabular}


TABLA 5.2

¿CuÁNTO UTILIZAS LaS Siguientes TECNOLOGÍas?

(\% SOBRE LOS QUE HAN CONTESTADO LA ENCUESTA)

\begin{tabular}{|l|c|c|c|c|c|c|}
\hline & \multicolumn{2}{|c|}{$\begin{array}{c}\text { Diseñar, "COLGAR" Y MANTENER UNA } \\
\text { WEB, Un BLOG, Un FOTOLOG } \ldots\end{array}$} & \multicolumn{3}{c|}{ CORREO ELECTRÓNICO (EMAIL) } \\
\hline & No-J & $\mathrm{J}$ & JM & No-J & J & JM \\
\hline Nunca & 36,1 & 44,8 & 40 & 9,8 & 25,4 & 17,1 \\
\hline Pocas veces & 15,7 & 19 & 20 & 26,8 & 27,5 & 31,4 \\
\hline A veces & 18,1 & 8,4 & 17,1 & 31,7 & 28,7 & 25,7 \\
\hline Muchas veces & 30,1 & 17,8 & 22,9 & 31,7 & 18,3 & 25,7 \\
\hline & 100 & 100 & 100 & 100 & 100 & 100 \\
\hline
\end{tabular}

Por lo tanto, con todas las cautelas necesarias dado el carácter exploratorio del estudio, las conclusiones de la encuesta parecen apuntar a que en este tema el País Vasco se nos presenta plenamente integrado en la Sociedad de Consumo Global. La mayoría de los adolescentes que han contestado la encuesta (el 80\%) utiliza los juegos electrónicos. Los chicos juegan más que las chicas, y los de 11 años más que los de 18. Sus videojuegos favoritos son los mismos que los de los adolescentes del resto del mundo: Pro Evolution Soccer, Grand Theft Auto (juego recomendado para mayores de 18 años), Mario Bros, Sims, Fifa, NBA... Es decir, nos encontramos ante un fenómeno que se presta a ser un buen ejemplo para las posturas que alertan del posible peligro de una excesiva homogenización cultural.

En lo que respecta al peligro de adicción que pueden suponer estos nuevos juegos, podríamos decir que el 10\% de los que han contestado juega "mucho" o "demasiado", porque ese es el porcentaje que dice jugar cuatro horas o más los días festivos. Pero en general no se confirma la hipótesis que define al videojugador con el estereotipo de "chico joven raro o "friki" que se pasa la vida aislado en su habitación, enganchado a los videojuegos violentos": la mayoría de jugadores tiene parecidas costumbres de ocio, y parecidas actitudes hacia las nuevas tecnologías que los no jugadores... Lo que por otra parte es lógico, porque una amplía mayoría (el 80\% de los que han contestado la encuesta) se autodeclara videojugadora. Sólo se aprecian algunas diferencias entre los que juegan mucho y el resto (con menos horas de lectura y menos tiempo con los amigos en el caso de los primeros). Es decir, no deberíamos demonizar el simple hecho de jugar con juegos electrónicos. Lo que hay que hacer es afinar los análisis y las investigaciones, diferenciando cuánto se juega y a qué se juega. Porque así como existen diferentes tipos de libros y de programas de televisión (programas que sólo entretienen, programas no recomendados para menores y programas educativos), también existen diferentes tipos de juegos digitales. Y lo mismo cabe decir sobre el tiempo de juego: afirmar que "jugar con videojuegos es una simple pérdida de tiempo" sería una generalización excesiva. En primer lugar, porque concretar y diferenciar la cantidad de tiempo empleado es importante (no es lo mismo jugar una hora al día que jugar cuatro). Y en segundo lugar, porque habría que analizar también a qué otras actividades se 
les está robando ese tiempo y qué juegos digitales se utilizan (desde un punto de vista educativo, jugar con un "juego digital serio" seguramente es mejor que ver un programa de "televisión basura").

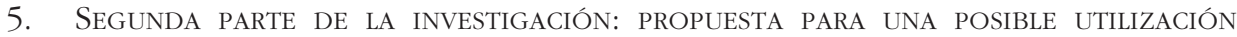
EDUCATIVA DE LOS VIDEOJUEGOS

El segundo objetivo de nuestra investigación ha consistido en diseñar y probar un experimento o taller para utilizar educativamente los videojuegos. Los objetivos concretos de este experimento han sido:

1) Plantear una prueba que posibilite el acercamiento entre la escuela y el mundo "real" de los adolescentes. En nuestro caso, se propone introducir en la escuela una de las pantallas más importantes en la cultura de los adolescentes (los videojuegos).

2) Empezar a trabajar una mirada reflexiva y crítica hacia los juegos digitales, objetivo que se enmarcaría en la línea de lo que se ha definido como "aprender a mirar pantallas" o "pedagogía de los medios". Así como en la cultura escrita ha sido necesario conocer, analizar y saber valorar los textos fundamentales de esa cultura, sería recomendable plantear lo mismo para las generaciones a las que les ha tocado vivir en las culturas audiovisual y digital.

3) Mostrar que con las nuevas tecnologías, con las pantallas y en nuestro caso concretamente con los juegos electrónicos también se pueden aprender "cosas interesantes y buenas" (en la línea de lo que se ha definido como "aprender con pantallas" o "pedagogía con los medios").

Teniendo en cuenta estos antecedentes, el primer esquema o guion que preparamos para nuestras sesiones fue el siguiente (Cuadro 1):

\section{Cuadro 1. Primer guion Para un taller educativo con Videojuegos}

1. Reflexión sobre los aspectos polémicos de los videojuegos. Análisis de videojuegos que fomentan contravalores (violencia, sexismo, racismo, etc.).

2. Reflexión sobre las posibles potencialidades de los videojuegos. Jugar a "videojuegos serios", para entender que así como existen diferentes programas de televisión, diferentes películas y diferentes libros, también hay que diferenciar los diferentes tipos de juegos.

3. Reflexión general sobre la sesión y sobre el fenómeno de los videojuegos.

Siguiendo este esquema, el siguiente paso consistió en elegir los videojuegos que queríamos utilizar en nuestras sesiones. Para la primera parte ("reflexión sobre los aspectos polémicos") elegimos los juegos Grand Theft Auto (GTA) y Bully porque permitían trabajar los objetivos que nos planteábamos: en el videojuego 
GTA ("Gran ladrón de coches"), el jugador, a través de su "avatar" o personaje en el juego, debe cumplir las misiones que se le asignan, misiones que podríamos definir como "polémicas" y "poco apropiadas para menores": repartir palizas, robar coches, conseguir droga... Además, mientras realiza estos "trabajos", puede hacer otras cosas por capricho: visitar prostíbulos, atropellar a la gente mientras conduce, etc. El videojuego Bully transcurre en una escuela, y el protagonista (el jugador) deberá utilizar la violencia contra el resto de alumnos (la palabra inglesa bully significa "miedo" cuando se trata de un verbo y "matón" cuando funciona como nombre). Se trata, por lo tanto, de un juego muy apropiado para lanzar el debate sobre el acoso escolar. Pero el problema con las últimas versiones del GTA (el GTA4) y el juego Bully es que de momento sólo se pueden jugar en consolas, y no hay consolas en las escuelas. Por lo tanto, para trabajar con estos juegos decidimos utilizar videos (grabaciones de partidas que anteriormente habíamos jugado nosotros o grabaciones que ya existían en youtube o en otras webs), a pesar de que de esta manera no podríamos aprovechar uno de los mayores atractivos de los videojuegos: la posibilidad de interactuar con el juego. Para el resto de la sesión, intentamos trabajar con videojuegos que se pudieran jugar en el ordenador: el GTA-San Andreas (una versión anterior de la saga GTA), Contra viento y marea (videojuego "serio" diseñado por las Naciones Unidas que nos ofrece la posibilidad de "vivir" la vida de los inmigrantes) y McDonalds (videojuego crítico diseñado por Molleindustria que pretende suscitar debate sobre la comida rápida y sobre el mundo de las grandes empresas). El guión definitivo quedaba por lo tanto de la siguiente manera (Cuadro 2):

\section{Cuadro 2. Guion definitivo para un taller educativo CON Videojuegos}

1. Presentación

2. Visionado de videos

2.1. Grand Theft Auto-4

2.2. Bully

3. Jugar con videojuegos

3.1. Grand Theft Auto-San Andreas

3.2. Contra viento y marea

3.3. McDonalds

4. Reflexión y debate sobre la sesión (primero en grupos pequeños, luego en grupo grande/clase).

Basándonos en este guion, se llevaron a cabo cuatro sesiones durante el otoño del 2008. Dos en la escuela Urola de Azpeitia (Gipuzkoa) y dos en la escuela Laskorain de Tolosa (Gipuzkoa). La duración de cada una de las dos primeras sesiones fue de una hora, trabajando con una clase de Tercero de Eso (alumnos de 14 años) y con otra clase de Primero de Bachiller (alumnos de 16 años). Se grabó un video de cinco minutos del videojuego GTA-4 con los momentos que nos interesaba ver (robo de coches, peleas, tratamiento sexista de la mujer...). Para el 
visionado de cinco minutos del videojuego Bully utilizamos youtube (en Internet podemos encontrar muchos videos de la mayoría de los juegos más conocidos que nos pueden servir para hacernos una primera idea). Después de ver estos videos, empezamos a jugar el GTA-San Andreas (para lo cual hay que cargar primero el juego en todos los ordenadores), y utilizamos Internet para los otros videojuegos (contravientoymarea.org y molleindustria.org/mcdonalds).

Los resultados de estas dos primeras sesiones fueron totalmente satisfactorios: la motivación de los alumnos fue muy alta, y se plantearon interesantes debates sobre el fenómeno de los videojuegos (sobre los comportamientos y valores polémicos que se pueden ver en algunos videojuegos, sobre los diferentes tipos de juegos, etc.). Invitamos también a los profesores y a la coordinadora Tic de la escuela que habían asistido a las sesiones a responder tres preguntas breves («¿Cuál es tu opinión sobre la sesión? ¿Cuáles son las posibilidades o las potencialidades de este tipo de sesiones? ¿Y las limitaciones?"), y las valoraciones fueron muy positivas, definiendo la propuesta como "muy interesante" ("para las clases de ética", "para hablar sobre valores", "para reflexionar sobre lo que ven"). En cuanto a los aspectos a mejorar para las siguientes sesiones, había que replantearse dos cuestiones: con respecto a la edad de los participantes, teniendo en cuenta los objetivos, la metodología y los juegos, la sesión parecía funcionar mejor con los mayores; en cuanto al tiempo, parecía necesario alargar la duración de las sesiones.

Por eso, en la tercera y cuarta sesión trabajamos con Primero y Segundo de Bachiller (alumnos de 16 y 17 años), y cada una de las sesiones pasó a tener una duración de dos horas. Estos cambios nos permitieron plantear mejor las sesiones, por ejemplo, pidiendo a los alumnos que despues de ver los videos o utilizar los juegos contestaran individualmente y por escrito a las siguientes preguntas (Cuadro 3).

Por lo tanto, en esta segunda parte de nuestra investigación hemos diseñado y probado unas sesiones para trabajar educativamente este fenómeno. Los adolescentes que han participado en las mismas han podido hablar y reflexionar sobre los videojuegos en clase, y han podido ver que existen diferentes tipos de videojuegos: juegos que fomentan lo que la sociedad ha definido como "contravalores" y juegos que además de entretener buscan la reflexión y la educación. Por ejemplo, ha funcionado muy bien en nuestros talleres el debate sobre "el sexismo en los videojuegos", planteándose fuertes discusiones entre las chicas que criticaban duramente la utilización de la mujer como simple "objeto sexual" en muchos juegos y algunos chicos que intentaban explicar/justificar por qué les gustaban estos juegos. Los alumnos que han participado en estos experimentos han reflexionado y hablado también sobre la violencia, sobre la globalización, sobre las diferencias entre el mundo real y el mundo virtual... Y hemos podido comprobar que no se cumple el mito que define como aburridos los videojuegos serios: aunque evidentemente los juegos comerciales tengan mejores gráficos y mejor "jugabilidad", los participantes en nuestras sesiones han reconocido que los juegos serios que proponíamos (Contra viento $y$ marea y McDonalds) les han parecido buenos y entretenidos. 


\section{Cuadro 3. Taller educativo con Videojuegos, CUESTIONARIO PARA LOS PARTICIPANTES}

1. ¿Qué te ha parecido el primer video $(G T A-4)$ ? ¿Qué tipo de comportamientos, valores, tipos de personas se pueden ver en el juego? ¿Qué tipo de tratamiento se da a la mujer, con qué temas se la relaciona?

2. ¿Qué puedes decir sobre el segundo video que hemos visto (Bully)? ¿Has notado alguna diferencia entre el "discurso oficial" (el de la escuela, la familia) y el discurso de este videojuego?

3. ¿Qué te ha parecido jugar a GTA-San Andreas? ¿Qué tipo de comportamientos, valores, tipos de personas se pueden ver en el juego?

4. ¿Qué dirías sobre el videojuego Contra viento y marea? ¿Te parece divertido? ¿Cuál es el tema del juego? ¿Y el objetivo general? ¿Tiene alguna relación con lo que sucede en el mundo real?

5. ¿Qué te ha parecido el juego McDonalds? ¿Te parece divertido? ¿Cuál es el tema del juego? ¿Y el objetivo general? ¿Con qué dificultades te has encontrado en el papel del dueño de la empresa? ¿Cómo has solucionado estos problemas? ¿Cuál es el objetivo de la empresa?

6. ¿Puedes apreciar diferencias entre los videojuegos que hemos utilizado? ¿Cuáles son esas diferencias?

En cuanto a las limitaciones de nuestra propuesta podríamos citar las siguientes: (1) Con la metodología y los juegos que hemos utilizado, a los más jóvenes les ha costado entender el objetivo de las sesiones. Existe, por lo tanto, el peligro de que algunos entiendan este tipo de ejercicios como "horas de recreo". Ante esto se nos ocurren dos posibles soluciones: por una parte, para trabajar con alumnos más jóvenes, habría que revisar la propuesta (repensar metodología y juegos); por otra parte, hay que explicar el objetivo de las sesiones («analizar el fenómeno de los videojuegos") y dar seriedad a la propuesta (recalcando que no van a realizar una sesión teórica, pero que tampoco se trata de simple entretenimiento; invitando a tener encima de la mesa papel y bolígrafo; leyendo las preguntas antes de ver los videos o de jugar para saber en qué tienen que fijarse; y dejándoles unos minutos tras cada juego para que escriban sus reflexiones). (2) La escuela, como institución, no parece preparada para este tipo de propuestas. Uno de los problemas con los que nos hemos encontrado ha sido, por ejemplo, el de las infraestructuras: escuelas de 1.000 alumnos con dos salas de ordenadores, cada una con 20 ordenadores (una para la Educación Primaria y la otra para la ESO y Bachillerato); además, en una de las escuelas los ordenadores no tenían lector de DVD, por lo que en vez de jugar al GTA-San Andreas tuvimos que verlo en video. Otro problema puede ser el del profesorado. En general, hemos percibido poco interés por parte de los profesores, hasta el extremo de que algunos prefirieron ausentarse antes que asistir a las sesiones (nos referimos a los profesores a los que les tocaba estar con esos grupos en esas horas). Y a nuestra propuesta de valorar las sesiones contestando tres breves preguntas sólo respondieron dos de los cinco profesores con los que hemos trabajado. Uno de los motivos de esta falta de interés puede ser la falta de formación: seguramente la mayoría de ellos veía muy lejano ese mundo (uno 
de los profesores no tenía ni siquiera dirección de correo electrónico), y hasta ahora seguramente no se les ha explicado el interés de este tipo de propuestas. Puede constituir también un problema la organización escolar: calendarios y horarios repletos de actividades y sin tiempo para incorporar nuevas propuestas, currículums anticuados, una amplia y quizás excesiva gama de exigencias que la escuela y los profesores supuestamente deberían responder...

\section{CONClusiones}

6.1. El de los videojuegos es un fenómeno social muy importante. Mueven mucho dinero, los utiliza mucha gente y son muy atractivos, porque, comparados con el resto de pantallas, ofrecen más posibilidades de participación o de interactividad.

6.2. En cuanto al primer objetivo de nuestro estudio, con todas las cautelas necesarias dado el carácter exploratorio de la investigación, los datos nos indican algunas tendencias que tendríamos que seguir analizando en posteriores trabajos: la mayoría de los adolescentes que han contestado la encuesta (el 80\%) se autodeclaran videojugadores, y no parece haber diferencias significativas entre jugadores y no jugadores en cuanto a hábitos de ocio y actitud y uso de TIC. Las diferencias existen entre los que juegan mucho (cuatro horas o más los días festivos) y el resto.

Estas diferencias nos ofrecen también algunas pistas para el debate sobre los peligros de los juegos digitales: los videojuegos no son peligrosos. Lo que puede resultar peligroso es jugar mucho o demasiado, tal como hace el 10\% de los adolescentes que ha contestado nuestra encuesta (el grupo de los que declara que los días festivos juegan cuatro horas o más). O lo que puede ser también peligroso es que los menores utilicen juegos que no son apropiados para su edad, tal como hace el $46 \%$ de los adolescentes que han contestado nuestra encuesta.

6.3. En el ámbito educativo (escuelas, familia, ocio...) puede ser interesante utilizar los videojuegos en un doble sentido: aprender a mirar pantallas y aprender con pantallas.

En cualquier caso, parece necesario algún tipo de intervención pública y educativa ante estas nuevas pantallas, porque, de lo contrario, estaríamos dejandolas en manos de la industria, para la cual, los debates ético-morales son menos importantes que la ganancia económica. 


\section{REFERENCIAS BIBLIOGRÁFICAS}

Adese, Asociación de Distribuidores y Editores de Software de Entretenimiento (2009) Usos y hábitos de los videojugadores españoles. Descargado el 15 de marzo de 2011. www.adese.es.

- (2010) Anuario 2009. Descargado el 7 de marzo de 2009. www.adese.es.

Amnistía Internacional (2004) Videojuegos: ¿qué valores transmiten? Descargado el 21 de noviembre de 2007. www.i-a.es.

Anderson, C. A.; Douglas, A. G. y Buckley, K. E. (2007) Violent video games effects on children and adolescents: theory, research, and public policy. Oxford, Oxford University Press.

Byron, T. (2008) Safer Children in a Digital World. The Report of the Byron Review. Descargado el 28 de febrero de 2009. www.dcsf.gov.uk/byronreview.

Camas, M. y Almazán, L. M. (2006) Jóvenes y videojuegos. Análisis de la relación que se establece entre los videojuegos y sus jóvenes usuarios: cuándo se empieza, a qué se juega, etc. Comunicación y Pedagogía, 216, 37-41.

Cassel, J. y Jenkins, H. (1998) From Barbie to Mortal Kombat: Gender and Computer Games. Cambridge-EE. UU., MIT Press.

De Freitas, S. (2008) Serious Virtual Worlds: a scoping study. Descargado el 28 de febrero de 2009. http://www.jisc.ac.uk/media/documents/publications/seriousvirtualworldsv1. pdf.

De Miguel, R. (2006) Cuestiones en torno al poderoso efecto de los videojuegos violentos: del neoconductismo a la cognición social. Icono 14 (en línea), 7. Descargado el 17 de febrero de 2007. www.icono14.net/revista.

Díez GuTiérRez, E. J. (2004) La diferencia sexual en el análisis de videojuegos. Madrid, Instituto de la Mujer-Ministerio de Trabajo y Asuntos Sociales y ciDe-Ministerio de Educación y Ciencia.

EcheberRía, J. (2008) Videojuegos: educación, socialización, en Videojuegos: educación, socialización, innovación. Curso de Verano de la Universidad del País Vasco. San Sebastián, 8-9 septiembre, Resúmenes de Ponencias, 10-15.

EgenfeldT-Nielsen, S. (2007) Educational Potential of Computer Games. Londres, Continuum.

Egenfeldt-Nielsen, S.; Smith, J. H. y Pajares Tosca, S. (2008) Understanding Video Games: The Essential Introduction. Londres, Routledge.

Elus, H. et al. (2006) Unlimited Learning: Computer and Video Games in the learning landscape. Descargado el 3 de febrero de 2008. http://www.elspa.com/assets/files/u/unlimitedlearningtheroleofcomputerandvideogamesint_344.pdf.

Estallo, J. A. (1995) Los videojuegos. Juicios y prejuicios. Barcelona, Planeta.

EtXeberría, F. (2006) ¿Son violentos los videojuegos violentos? Comunicación y Pedagogía, 216, 26-32.

- (2008) El consumo de los videojuegos: implicaciones educativas, en Videojuegos: educación, socialización, innovación. Curso de Verano de la Universidad del País Vasco. San Sebastián, 8-9 septiembre. Resúmenes de Ponencias, 15-20.

Ferrer, M. y Ruiz, J. A. (2005) Uso de videojuegos en niños de 7 a 12 años. Una aproximación mediante encuesta. Icono 14 (en línea), 7. Descargado el 17 de febrero de 2007. www.icono14.net/revista.

Freedman, J. L. (2002) Media violence and its effect on aggression: assessing the scientific evidence. Toronto, University Toronto Press. 
GeE, J. P. (2004) Lo que nos enseñan los videojuegos sobre el aprendizaje y el alfabetismo. Málaga-Granada, Aljibe-Consorcio "Fernando de los Ríos" para la enseñanza abierta en Andalucía.

- (2007) Good Video Games and Good Learning: Collected Essays on Videos Games, Learning and Literacy. Nueva York, Peter Lang Publishing.

Gros, B. (coord.) (1998) Jugando con videojuegos: educación y entretenimiento. Bilbao, Desclée de Brouwer.

- (2004) Pantallas, juegos y educación. La alfabetización digital en la escuela. Bilbao, Desclée de Brouwer.

- (2008) Videojuegos y aprendizaje. Barcelona, Graó.

Grossman, D. y De Gaetano, G. (1999) Stop teaching and kids to kill: a call to action against TV, movie and video games violence. Nueva York, Grown.

Jenkins, H. (2006) Media education for the $21^{\text {st }}$ Century. Descargado el 23 de febrero de 2009. www.MacFound.org.

JONES, G. (2002) Killing monsters: why children need fantasy, super heroes, and make-believe violence. Nueva York, Basic Books.

Kane, J.; Middaugh, J. y Evans, C. (2008) Teens, Video Games and Civics. Descargado el 23 de febrero de 2009. www.MacFound.org.

KutNer, L. y Olson, C. (2008) Grand Theft Childhood: the surprising truth about violent video games and what parents can do. Londres, Simon \& Schuster.

LACASA, P. et al. (2007a) Classrooms as 'living labs': the role of commercial games, en The $5^{\text {th }}$ Media in Transition Conference. Massachusetts Institute of Technology (MIT), 27-29 abril. Descargado el 16 de enero de 2009. www.web.mit.edu.

- (2007b) Aprendiendo con los videojuegos. Descargado el 21 de febrero de 2010. www. aprendeyjuegaconea.com.

- (2009) Videojuegos en el Instituto: Ocio digital como estímulo en la enseñanza. Descargado el 21 de febrero de 2010. www.aprendeyjuegaconea.com.

Levis, D. (1997) Los videojuegos, un fenómeno de masas. Qué impacto produce sobre la infancia y la juventud la industria más próspera del sistema audiovisual. Barcelona, Paidós.

Manders, T. (2008) Draft Report on the protection of consumers, in particular minors, on respect of the use of video games. Descargado el 21 de marzo de 2010. http://europarl. europa.eu.

Provenzo, E. (1991) Video Kids: Making sense of Nintendo. Cambridge-Ee. uU., Harvard University Press.

Rodríguez SAn Julián, E. (coord.) (2002) Jóvenes y videojuegos. Espacio, significación y conflicto. Madrid, Fundación de Ayuda contra la Drogadicción e Injuve.

Sanford, R. y Williamson, B. (2005) Games and Learning. Descargado el 3 de febrero de 2008. http://www.futurelab.org.uk/resources/documents/handbooks/games_and_ learning2.pdf.

SHAFFer, D. W. (2008) How computer games help children learn. Nueva York, Palgrave Mcmillan. 